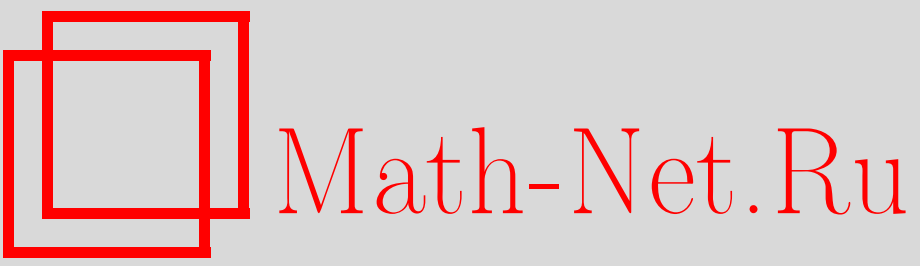

А. И. Кириллов, В. Ю. Мамакин, Стохастическая модель фазового перехода и метастабильность, ТMФ, 2000, том 123, номер 1, 94-106

DOI: https://doi.org/10.4213/tmf589

Использование Общероссийского математического портала Math-Net.Ru подразумевает, что вы прочитали и согласны с пользовательским соглашением

http://www.mathnet.ru/rus/agreement

Параметры загрузки:

IP: 3.81 .55 .215

26 апреля 2023 г., 05:10:18 
ТЕОРЕТИЧЕСКАЯ

И МАТЕМАТИЧЕСКАЯ

ФИЗИКА

Том 123, № 1

апрель, 2000

(C) $\mathbf{2 0 0 0}$ г.

А.И. Кириллов* , В. Ю. Мамакин ${ }^{\dagger}$

\section{СТОХАСТИЧЕСКАЯ МОДЕЛЬ ФАЗОВОГО ПЕРЕХОДА И МЕТАСТАБИЛЬНОСТЬ}

Эволюция системы, в которой может произойти фазовый переход, моделируется марковским процессом с матрицей переходных вероятностей, зависящей от параметра. При изменении этого параметра изменяется стационарное распределение марковского процесса, что интерпретируется как фазовый переход системы из одного термодинамически равновесного состояния в другое. Вычисления и компьютерные эксперименты проведены применительно к конденсации пара. Выборочные траектории марковского процесса имеют участки с приблизительно постоянными значениями радиуса конденсирующихся капель. Эти участки проявляются как метастабильные состояния. Обнаружено два метастабильных состояния: начальное (перегретый пар) и промежуточное (туман). Оценены вероятностные распределения радиусов капли в метастабильных состояниях.

\section{1. ВВЕДЕНИЕ}

Мы исследуем системы, которые могут находиться в одном из двух состояний $A$ или $B$ в зависимости от значения некоторого параметра $\theta$. Типичным примером $\theta$ является температура. Предполагается, что состояние $A$ устойчиво, а состояние $B$ неустойчиво, если $\theta$ меньше некоторой величины $\theta_{\mathrm{cr}}^{A}$, и состояние $B$ устойчиво, а состояние $A$ неустойчиво, если $\theta$ больше некоторой величины $\theta_{\mathrm{cr}}^{B}$. Очевидно, что $\theta_{\mathrm{cr}}^{A} \leqslant \theta_{\mathrm{cr}}^{B}$.

Цель статьи - описать переход системы из состояния $A$ в состояние $B$ в результате того, что значение параметра $\theta$ было быстро увеличено с $\theta=\theta_{1}<\theta_{\mathrm{cr}}^{A}$ до $\theta=\theta_{2}>\theta_{\mathrm{cr}}^{B}$. Такие переходы называются фазовыми. Их примерами служат:

1. Вскипание жидкости. A- жидкое состояние, $B$ - газообразное состояние, $\theta$ температура, $\theta_{\text {cr }}^{A}$ и $\theta_{\text {cr }}^{B}$ - границы температурной устойчивости жидкости и пара при фиксированных значениях остальных термодинамических параметров.

2. Конденсация пара. A-газообразное состояние, $B$ - жидкое состояние, $\theta$ - обратная температура, так что увеличение $\theta$ соответствует понижению температуры, $\theta_{\mathrm{cr}}^{A}$ и $\theta_{\mathrm{cr}}^{B}$ - границы температурной устойчивости жидкости и пара при фиксированных значениях остальных термодинамических параметров.

\footnotetext{
* Высший колледж математической физики, Независимый московский университет, Москва, Россия; Московский энергетический институт, Москва, Россия. E-mail: tmph@mi.ras.ru

${ }^{\dagger}$ Высший колледж математической физики, Независимый московский университет, Москва, Россия; Московский государственный университет, Москва, Россия. E-mail: vmamakin@orc.ru
} 
3. Плавление твердого тела. $A$ - кристаллическое состояние, $B$ - жидкое состояние, $\theta$ - температура. Пусть $T_{1}$ и $T_{2}$ - температуры плавления и кристаллизации. Возможно, что $T_{1}>T_{2}$. Поэтому мы примем, что $\theta_{\mathrm{cr}}^{A}=\min \left\{T_{1}, T_{2}\right\}$ и $\theta_{\mathrm{cr}}^{B}=\max \left\{T_{1}, T_{2}\right\}$.

4. Возникновение спонтанной намагниченности. $A$-состояние решетки спинов с нулевым магнитным моментом, $B$ - состояние с ненулевым магнитным моментом, $\theta-$ обратная температура, $\theta_{\mathrm{cr}}^{A, B}=T_{\mathrm{C}}^{-1}$, где $T_{\mathrm{C}}$ - температура Кюри.

5. Переориентация спинов решетки при изменении внешнего магнитного поля $H$. $A$-состояние, при котором большинство спинов направлено вниз, $B$ - состояние, при котором большинство спинов направлено вверх, $\theta=H$. Если температура ниже критической, то $\theta_{\mathrm{cr}}^{A, B}= \pm\left|H_{\mathrm{cr}}\right|$.

Существуют и другие фазовые переходы, аналогичные указанным выше, к описанию которых применимы методы этой статьи.

Мы предполагаем, что ансамбль систем в каждый момент времени полностью характеризуется вектором $X(t)$ некоторого пространства $\mathcal{X}$. Очевидно, что $X(t)$ - случайный вектор. Если система находится в термодинамическом равновесии, то векторы $X(t)$ имеют одно и то же вероятностное распределение во все моменты времени $t$. Это вероятностное распределение называется в статистической физике состоянием или мерой Гиббса.

По каждой вероятностной мере можно построить стационарный случайный процесс, значения которого в каждый момент времени распределены по закону, определяемому данной вероятностной мерой. Поэтому вместо мер Гиббса состояния равновесных систем можно описывать стационарными случайными процессами. В данной работе мы предполагаем, что соответствие $t \mapsto X(t)$ - случайный процесс, не только если система находится в равновесии, но и в остальных случаях.

Идея использовать случайные процессы вместо мер не нова. Она лежит в основе метода стохастического квантования, предложенного Паризи и Ву [1] для построения мер Нельсона в евклидовой квантовой теории поля. Позднее этот метод был обоснован в работах [2] для конкретных моделей. В математике он использовался для построения мер в работе [3]. В [4] был предложен новый подход к теории метастабильных состояний, основанный на описании ансамблей систем с помощью случайных процессов. В данной работе мы развиваем этот подход, распространяя его на фазовые переходы.

Заметим, что исходным пунктом стохастического квантования является так называемое уравнение Ланжевена

$$
d X(t)=K(X(t)) d t+d w(t)
$$

где $w(\cdot)$ - стандартный винеровский процесс со значениями в $\mathcal{X}$. Таким образом, в стохастическом квантовании $\mathcal{X}$ - континуум. Этого нельзя предполагать в теории фазовых переходов, т.к. у целого ряда систем конфигурационные пространства дискретны. Следовательно, процесс $X(\cdot)$ вовсе не обязательно должен быть решением уравнения типа (1). Мы предполагаем лишь, что $X(\cdot)$ - марковский процесс, и определяем его переходными вероятностями, а не стохастическим уравнением. 
Статья организована следуюшим образом. В разделе 2 описана стохастическая модель фазового перехода и приведены результаты численного моделирования случайного процесса $X(\cdot)$. Показано, что переход $A \mapsto B$ содержит один или несколько этапов, которые естественно назвать метастабильными состояниями. Статистические свойства метастабильного состояния описаны в разделе 3 . Оно не является состоянием в смысле статистической физики, но в разделе 3 мы показываем, что ему можно сопоставить аналог меры Гиббса. Раздел 4 содержит обсуждение полученных результатов.

\section{2. СТОХАСТИЧЕСКАЯ МОДЕЛЬ ФАЗОВОГО ПЕРЕХОДА}

2.1. Основной случайный процесс $X(\cdot)$. Микроскопические параметры системы, например координаты и импульсы молекул, образуют вектор, зависящий от времени $t$. Ансамбль статистически эквивалентных систем характеризуется случайным вектором микроскопических параметров. Обозначим этот вектор символом $X(t)$. Мы предполагаем, что при всех $t$ выборочные значения случайного вектора $X(t)$ принадлежат некоторому пространству $\mathcal{X}$. Поэтому при каждом $t$ вероятностное распределение случайного вектора $X(t)$ определяется некоторой вероятностной мерой $\mu_{t}$ на $\mathcal{X}$. Если системы ансамбля находятся в термодинамическом равновесии, то мера $\mu_{t}$ не изменяется с течением времени (она является мерой Гиббса). Однако случайный вектор $X(t)$ эволюционирует в любом случае, и возникает вопрос, можно ли трактовать эволюцию $X(t)$ как случайный процесс. Иными словами, можем ли мы, хотя бы в принципе, определить вероятностные распределения совокупностей случайных векторов $\left\{X\left(t_{1}\right)\right.$, $\left.X\left(t_{2}\right), \ldots, X\left(t_{n}\right)\right\}$ при всевозможных $n$ и $t_{1}<t_{2}<\cdots<t_{n}$. Если квантовые эффекты сушественны, то ответ на этот вопрос отрицательный, т.к. измерение $X\left(t_{1}\right)$ оказывает неконтролируемое воздействие на систему и результат измерения $X\left(t_{2}\right)$ будет зависеть от условий измерения $X\left(t_{1}\right)$, а не только от динамики системы, как в классической физике. Сказанное не означает, что вообще не сушествует случайного процесса $\widetilde{X}(\cdot)$ такого, что вероятностное распределение вектора $\widetilde{X}(t)$ равно заданной мере $\mu_{t}$. Например, стохастическая механика Нельсона [5] оперирует со случайным процессом, значение которого в каждый момент времени имеет плотность распределения $|\psi(t, x)|^{2}$, где $\psi$ - волновая функция системы. Однако физический смысл этого процесса еще не выяснен (см., например, [6]).

В данной статье мы предполагаем, что $X(\cdot)$ - случайный процесс. Его выборочные траектории описывают эволюции систем ансамбля. Поскольку случайный процесс неоднозначно определяется вероятностными распределениями $\mu_{t}$, мы можем выдвигать различные гипотезы о динамике случайного процесса $X(\cdot)$. Естественно предположить, что если системы ансамбля находятся в термодинамическом равновесии, то случайный процесс $X(\cdot)$ стационарен. Переходы между различными состояниями термодинамического равновесия мы описываем нестационарными процессами.

Если число компонент вектора $X(\cdot)$ велико, то мы вряд ли сможем найти их вероятностное распределение. Да и практическая польза такого распределения была бы невелика. К счастью, обычно существует некоторое небольшое число переменных $\xi_{1}, \ldots, \xi_{n}$, вероятностное распределение которых полностью характеризует состояние ансамбля. 
Рассмотрим, например, решетку из $N$ атомов со спином $s$. Конфигурация такой решетки полностью определяется $N$ переменными, каждая из которых может принимать только значения $-s,-s+1, \ldots, s$. С другой стороны, состояние ансамбля решеток определяется вероятностным распределением каких-нибудь $2 s$ переменных из $\xi_{-s}, \xi_{-s+1}, \ldots, \xi_{s}$, где $\xi_{j}(j=-s,-s+1, \ldots, s)-$ количество атомов, проекция спинов которых на ось $O z$ равна $j$. (Очевидно, что $\xi_{-s}+\xi_{-s+1}+\cdots+\xi_{s}=N$.)

Чтобы не обременять изложение непринципиальными усложнениями, возникаюшими, если размерность пространства $\mathcal{X}$ больше единицы, далее мы рассматриваем только конденсацию пара, поскольку состояние пара как ансамбля капель характеризуется вероятностным распределением единственного параметра - массы капли. В данном случае пространство $\mathcal{X}$ одномерно и выборочное значение случайного вектора $X(t)$ - это масса какой-то из капель. Заметим, что пространство $\mathcal{X}$ одномерно и у ансамблей других систем, перечисленных во введении, кроме решеток атомов со спином больше $1 / 2$.

Другой подход к описанию процесса формирования капель можно найти в [7].

2.2. Марковские переходные вероятности. Мы предполагаем, что $X(\cdot)$ - однородный марковский процесс. В частности, $X(\cdot)$ может быть решением уравнения (1).

Процесс $X(\cdot)$ определяется марковскими переходными вероятностями. Их удобно исследовать в дискретном приближении, т.е. когда $t$ принимает значения $0, \tau, 2 \tau, \ldots$ $(\tau>0)$ и пространство $\mathcal{X}$ имеет конечное число элементов.

Выберем в $\mathcal{X}$ некоторую конечную решетку $\widetilde{\mathcal{X}}$ и занумеруем ее узлыв порядке возрастания (напомним, что $\mathcal{X}$ - одномерное пространство). Процесс $X(\cdot)$ аппроксимируется марковской цепью на решетке $\widetilde{\mathcal{X}}$. Обозначим символом $p_{i, j}$ вероятность перехода процесса $X(\cdot)$ из окрестности узла с номером $i$ в окрестность узла с номером $j$ за время $\tau$. Например, для решений уравнения (1)

$$
p_{i, j} \approx \frac{h}{\sqrt{2 \pi \tau}} e^{-[(j-i) h-K(i h) \tau]^{2} /(2 \tau)},
$$

где $h$ - шаг решетки $\widetilde{\mathcal{X}}$, причем $h \sim \sqrt{\tau}$.

Из (2) следует, что

$$
p_{i, i} \approx \frac{h}{\sqrt{2 \pi \tau}}
$$

и

$$
p_{i, j} \approx \frac{h}{\sqrt{2 \pi \tau}} e^{-(j-i)^{2} \frac{h^{2}}{2 \tau}}[1+(j-i) K(i h) h] .
$$

Эти формулы показывают, что если предположить, что $p_{i, j}>0$ только при $j=i-1$, или $j=i$, или $j=i+1$, то получится неплохое приближение.

Поскольку $p_{i, i-1}+p_{i, i}+p_{i, i+1}=1$, только две переходные вероятности из трех можно выбирать произвольно. Условимся изображать матрищу переходных вероятностей $\left(p_{i, j}\right)$ совокупностью векторов $\left\{p_{i, i}, p_{i, i+1}-p_{i, i-1}\right\}$, приложенных к узлам решетки $\widetilde{\mathcal{X}}$ на вертикальной прямой (рис. 1 ).

Заметим, что проекция вектора $\left\{p_{i, i}, p_{i, i+1}-p_{i, i-1}\right\}$ на вертикальную ось, т.е. $p_{i, i+1}-$ $p_{i, i-1}$, есть математическое ожидание прирашения $X(t+\tau)-X(t)$ при условии, что

4 Теоретическая и математическая физика, т. 123, № 1, 2000 г. 


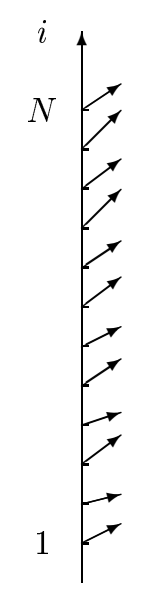

Рис. 1. Графобическое представление матрицы $p_{i, j}$.

$X(t)=h i$. Кроме того, вектор $\left\{p_{i, i}, p_{i, i+1}-p_{i, i-1}\right\}$ показывает вероятное направление изменения процесса $X(t)$.

Матрищу переходных вероятностей можно вычислить, например, следуюшим образом.

Слагаемое $K(X(t)) d t$ в уравнении (1) определяет среднее значение прирашения $d X(t)$. При конденсации пара приращение массы капли $X(t)$ пропорционально площади ее поверхности. Поэтому естественно принять, что $K(X(t)) \sim X(t)^{2 / 3}$. Если $\theta>\theta_{\mathrm{cr}}$, то капля имеет тенденшию к росту и наоборот. Поэтому

$$
K(X(t))=\alpha\left(\frac{\theta}{\theta_{\mathrm{cr}}}-1\right) X(t)^{2 / 3},
$$

где $\alpha$ - некоторое положительное число. Используя это равенство и $(2)$, получаем искомые переходные вероятности.

2.3. Зависимость переходных вероятностей от $\theta$. При температуре вьше критической вероятность $p_{i, i-1}$ испарения капли больше вероятности $p_{i, i+1}$ роста капли при всех значениях индекса $i$. Поэтому все векторы $\left\{p_{i, i}, p_{i, i+1}-p_{i, i-1}\right\}$ при высоких температурах направлены вниз, т.е. капли имеют тенденцию к уменьшению (рис. 2, область около $T=400 \mathrm{~K})$.

При температурах ниже критической температуры фазового перехода $p_{i, i+1}>p_{i, i-1}$; поэтому при низких температурах векторы $\left\{p_{i, i}, p_{i, i+1}-p_{i, i-1}\right\}$ направлены вверх, т.е. капли имеют тенденцию к росту (см. рис. 2 , область около $T=350 \mathrm{~K}$ ). При понижении температуры (т.е. росте $\theta$ ) значение вероятности $p_{i, i+1}$ увеличивается, а значение вероятности $p_{i, i-1}$ уменьшается. Соответственно векторы $\left\{p_{i, i}, p_{i, i+1}-p_{i, i-1}\right\}$ поворачиваются против часовой стрелки. Однако скорости поворота при различных значениях $i$ различны: векторы с большими значениями $i$ поворачиваются быстрее, чем векторы 


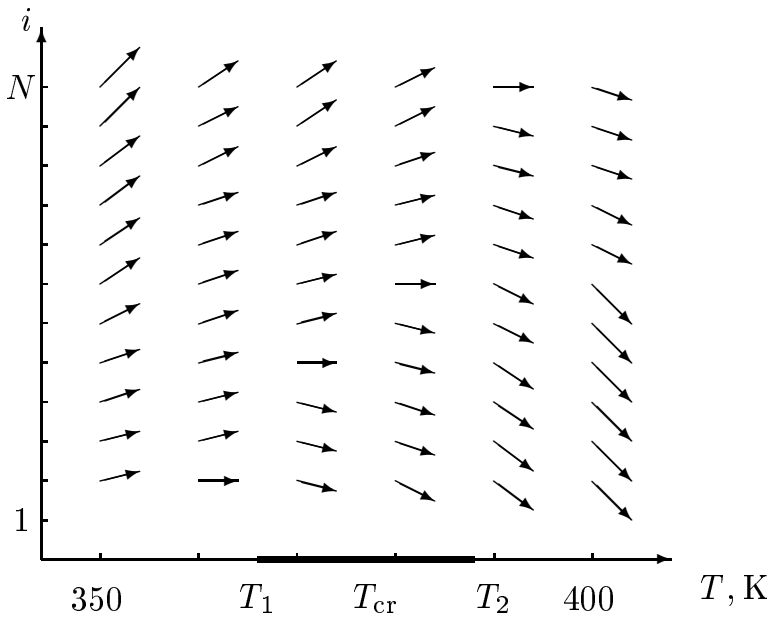

Рис. 2. Зависимость переходных вероятностей от температуры.

с малыми значениями $i$ (см. (5)). Поэтому сушествует интервал температур $\left[T_{1}, T_{2}\right]$, в пределах которого вероятность роста крупных капель больше вероятности их испарения (векторы направлены вверх), а для мелких капель - наоборот. Этот интервал температур на рис. 2 выделен жирной линией. Важно иметь в виду, что температура фазового перехода лежит в интервале $\left[T_{1}, T_{2}\right]$, т.к. состояние $A$ неустойчиво при $T_{1}<T<T_{2}$.

Формулы (3)-(5) подсказывают, что $p_{i, i}$ от $i$ не зависит, а

$$
p_{i, i \pm 1} \approx a\left(1 \pm b i^{2 / 3}\right),
$$

где $a>0$ и $b>0$ при $\theta>\theta_{\mathrm{cr}}$. Следовательно, величина $p_{i, i+1}$ растет с ростом $i$, а ве-

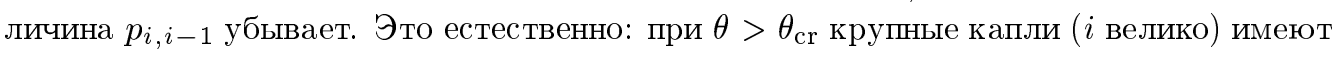

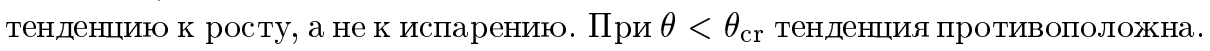

2.4. Свойства выборочных траекторий. Предположим, что системы ансамбля длительное время находились в условиях, при которых $\theta<\theta_{\mathrm{cr}}^{A}$. Тогда независимо от способа "приготовления" ансамбля $X(\cdot)$ станет стационарным процессом с вероятностным распределением случайного вектора $X(t)$, не зависящим от $t$ и соответствующим состоянию $A$. Обозначим это вероятностное распределение символом $\mu^{A}$. Если условия изменились так, что значение $\theta$ превысило $\theta_{\mathrm{cr}}^{B}$, то по истечении некоторого времени $X(\cdot)$ вновь станет стационарным процессом с вероятностным распределением случайного вектора $X(t)$, опять-таки не зависящим от $t$ и соответствуюшим состоянию $B$. Обозначим это вероятностное распределение символом $\mu^{B}$. Переход $X(\cdot)$ от стационарного процесса с распределением $\mu^{A}$ к стационарному процессу с распределением $\mu^{B}$ называется фазовым переходом $A \mapsto B$.

В наших экспериментах $N=100$ (число 100 выбрано условно). Начальное состояние $A$ задается стационарным распределением марковской цепи $p_{i}^{*}$ при $\theta<\theta_{\mathrm{cr}}$. Марковская цепь моделировалась стандартным способом. 
В состоянии $A$ (пар) $X(t) \approx 0$, а в состоянии $B$ (жидкость) $X(t) \approx 100$. Если параметр $\theta$ скачком изменяется от значения $\theta_{1}<\theta_{\text {cr }}^{A}$ до значения $\theta_{2}>\theta_{\text {cr }}^{B}$, то выборочные траектории покидают окрестность $X(t) \approx 0$ и переходят в окрестность $X(t) \approx 100$. Типичное поведение выборочных траекторий показано на рис. 3.

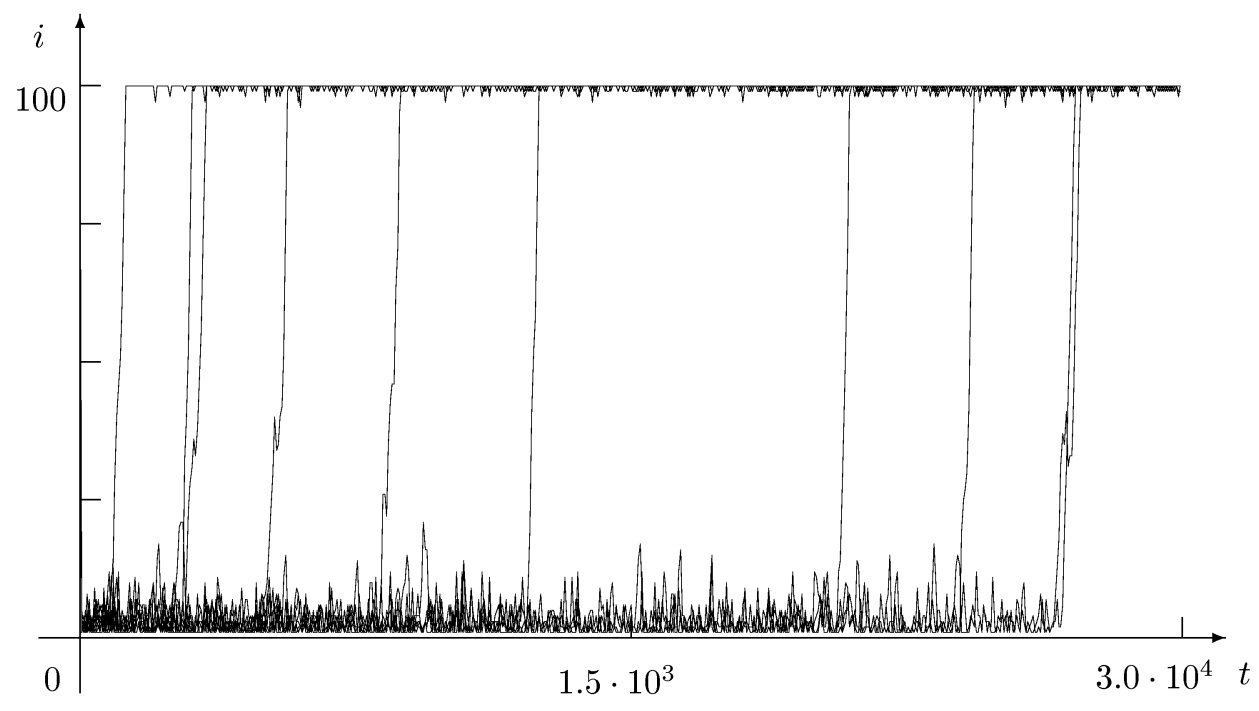

Рис. 3. Примеры выборочных траекторий роста капель.

Видно формирование десяти капель. Заметим, что при скачкообразном изменении параметра $\theta$ в момент $t=0$ каждая выборочная траектория некоторое случайное время находится в окрестности $X(t) \approx 0$, что с макроскопической точки зрения воспринимается как пребывание соответствуюшей системы в состоянии $A$ (переохлажденный пар), хотя это состояние уже не является устойчивым, поскольку $\theta>\theta_{\mathrm{cr}}^{B}$. Следуя [4], мы называем метастабильным состоянием этап фазового перехода, характеризуемый тем, что выборочные траектории в течение длительного времени не покидают некоторую малую область пространства $\mathcal{X}$. Для выборочных траекторий на рис. 3 таковой является область $X(t)<20$. Очевидно, что время пребывания в ней выборочных траекторий - случайная величина с выборочными значениями, равными примерно $10^{3}, 2.5 \cdot 10^{3}, 2.5 \cdot 10^{3}$, $5 \cdot 10^{3}, 7 \cdot 10^{3}, 13 \cdot 10^{3}, 22 \cdot 10^{3}, 24 \cdot 10^{3}, 27 \cdot 10^{3}$ и $27 \cdot 10^{3}$. Наша модель конденсации пара предсказывает сушествование еше одного метастабильного состояния в области $X(t)>20$. Это метастабильное состояние мы называем промежуточным. Его естественным аналогом является туман.

2.5. Этапы фазового перехода. На рис. 4 приведена типичная траектория образования капли. Видно, что формирование капли происходит в несколько этапов:

1. При температурах ниже критической состояние $A$ неустойчиво. Тем не менее, поскольку при малых размерах капли вероятность испарения выше вероятности конденсации, значительную часть времени процесс $X(t)$ находится в окрестности состояния с $i \approx 1$, т.е. с $i<i^{\prime}$, где $i^{\prime} \approx 20$, как видно на рис. 3 и 4 . 


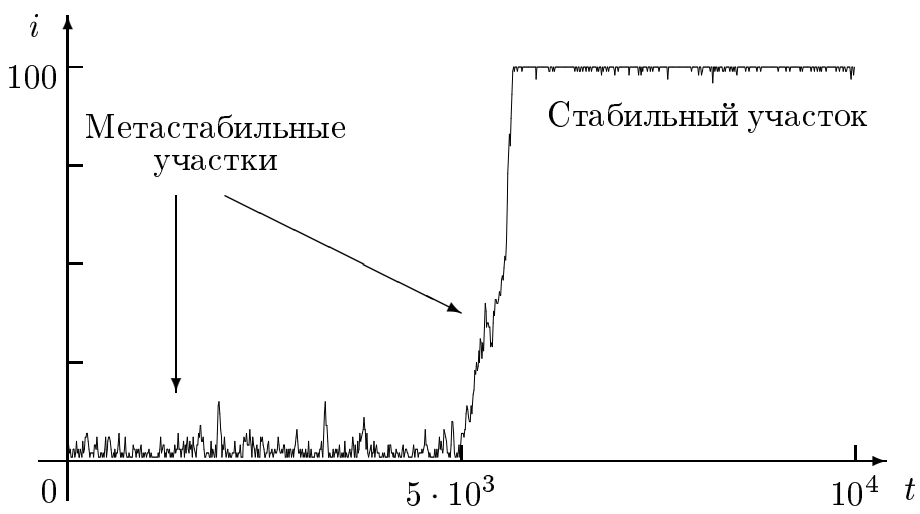

Рис. 4. Типичная траектория образования капли.

Численные эксперименты показывают, что после скачкообразного изменения температуры капля образуется за $3 \cdot 10^{4}$ шагов компьютерного времени с вероятностью 0.5.

Компьютерные эксперименты позволяют построить новое вероятностное распределение на траекториях, которые за определенное время не пересекли уровень $i=i^{\prime}$. Результаты численного моделирования показывают, что те траектории процесса $X(t)$, которые пребывают в зоне ожидания, очень быстро (примерно за $5 \cdot 10^{2}$ шагов) уподобляются траекториям стационарного процесса $X^{\prime}(t)$, определяемого матрищей перехода $P^{\prime}=P$ при $i, j<i^{\prime}$. Точнее говоря, вероятности $p_{i}=\operatorname{Prob}(X(t)=i)$, определяемые по траекториям, находящимся в зоне ожидания, практически не зависят от времени; и в сериях из $10^{3}, 10^{4}$ и $10^{6}$ испытаний было установлено, что $\sigma\left(p_{i}-p_{i}^{\prime}\right) \sim n^{-1}$, где $p_{i}^{\prime}-$ вероятности для стационарного распределения с матрицей $P^{\prime}$ и $n$ - число испытаний.

2. Покинув зону ожидания $X(t)<20$, траектории попадают в промежуточную зону $20<X(t)<80$. В этой зоне эволюция массы капли подобна случайному блужданию на прямой. Некоторые траектории задерживаются в промежуточной зоне, что видно на рис. 3 и 4 . Такие траектории уподобляются траекториям стационарного случайного процесса $X^{\prime \prime}(t)$ на промежутке от 20 до 80. Распределение процесса $X^{\prime \prime}(t)$ - это второе метастабильное состояние ансамбля капель конденсирующего пара. Эти участки траекторий подобны “экскурсиям за высокий барьер”, описанным в работе [8].

3. Траектория процесса может выйти из промежуточной зоны либо в зону ожидания $X(t)<20$, либо в зону $X(t)>80$. Если траектория процесса вернулась в зону ожидания, то капля не формируется, и опять требуется в среднем $3 \cdot 10^{4}$ шагов до очередного попадания в промежуточную зону. Неудачные попытки образования капли можно увидеть на рис. 3 (несколько мелких пиков, достигающих значений $20-30$ ).

Если траектория процесса попала в зону $X(t)>80$, то она быстро переходит в состояние с $i \approx 100$, т.е. формируется капля.

2.6. Понятие метастабильного состояния. В [4] было предложено интерпретировать метастабильные состояния как приблизительно стационарные участки на выборочных траекториях нестационарных процессов. В данной работе мы уточняем понятие 
приблизительной стационарности, предлагая считать, что пребывание системы в метастабильном состоянии описывается участком траектории нестационарного процесса со следующими свойствами:

а) траектория посешает не все состояния $\left\{X_{1}, \ldots, X_{N}\right\}$, а лишш некоторое их подмножество $\left\{X_{l}, \ldots, X_{m}\right\}$;

б) количество переходов траектории между состояниями $\left\{X_{1}, \ldots, X_{m}\right\}$ много больше числа состояний $\left\{X_{l}, \ldots, X_{m}\right\}$;

в) время пребывания траектории в области $\left\{X_{1}, \ldots, X_{m}\right\}$ много больше времени перехода из этой области в стабильную область.

На рис. 4 видны два метастабильных состояния. Другие определения метастабильного состояния даны в $[9,10]$.

\section{3. ИЗУЧЕНИЕ ПРОМЕЖУТОЧНОГО МЕТАСТАБИЛЬНОГО СОСТОЯНИЯ}

3.1. Описание численного эксперимента. При конденсации пара происходит около $10^{32}$ параллельных запусков процессов образования капли. При проведении столь же большого числа численных экспериментов мы смогли бы набрать много данных о промежуточных метастабильных участках типа того, который виден на рис. 4 . Поскольку не существует вычислительных средств, которые бы позволили провести $10^{32}$ экспериментов, мы увеличили вероятность появления промежуточного метастабильного участка. Для этого мы увеличиваем участок, на котором $p_{i, i+1}=p_{i, i-1}$ (т.е. стрелка горизонтальна), с одной точки (как на рис. 2) до множества $\left[i^{\prime}, \ldots, i^{\prime \prime}\right]$ (рис. 5). В наших численных экспериментах $i^{\prime \prime}=80$.

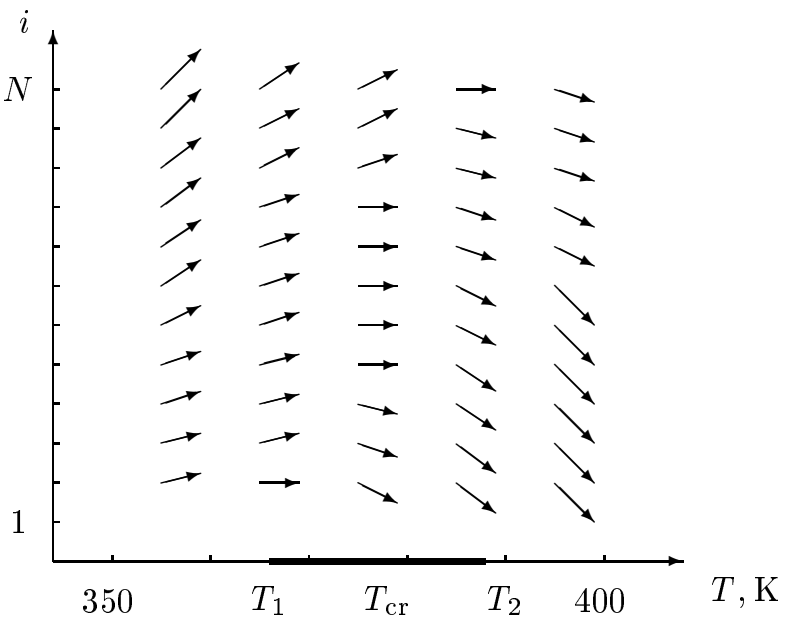

Рис. 5. Зависимость переходных вероятностей от температуры.

Для накопления достаточной статистики по промежуточному метастабильному состоянию мы запускали процессы непосредственно из точки $i=20$, чтобы не терять вре- 
мени на ожидание. Как было указано в разделе 2 , по достижении промежуточной зоны капля может сформироваться или не сформироваться. Поскольку мы интересуемся метастабильной фазой, которая является промежуточной между газообразным и жидким состояниями, мы учитываем только те траектории, которые заканчивались формированием капли без возвращения в зону ожидания.

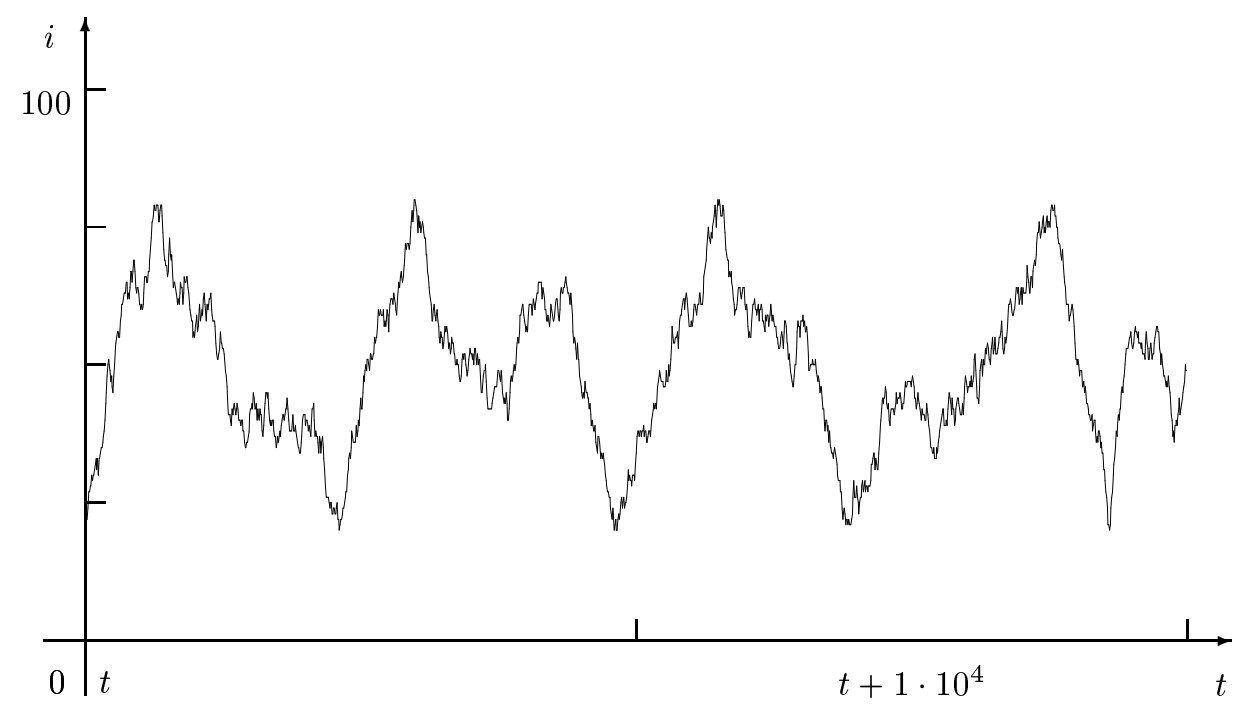

Рис. 6. Типичная часть выборочной траектории на промежуточном метастабильном участке (переходные вероятности, как на рис. 5).

В компютерном эксперименте путем последовательных запусков процессов из точки $X(0)=20$ наблюдалось $2 \cdot 10^{4}$ траекторий с образованием капли и более $6 \cdot 10^{5}$ возвращений в зону ожидания.

Среднее время жизни капли в промежуточной метастабильной фазе (т.е. среднее число шагов, которое было необходимо для достижения состояния $N=81$ ) составило 1600 шагов, максимальное - 12750 , минимальное - 203, $\sigma=1014$.

3.2. Эквивалентный стационарный процесс. Мы наблюдали $2 \cdot 10^{4}$ участков траекторий типа, изображенного на рис. 6 . Последовательно присоединяя один такой участок к другому с возможным отражением относительно прямой $i=50$, мы получили непрерывную зигзагообразную линию $x(t)$. Интерпретируя эту линию как выборочную траекторию некоторого процесса $X^{*}(t)$, мы установили, что эта траектория стационарна в том смысле, что для любой непрерывной ограниченной функции $f(x)$ интеграл

$$
\frac{1}{t_{2}-t_{1}} \int_{t_{1}}^{t_{2}} f(x(t)) d t
$$

имеет одно и то же значение при всевозможных $t_{1}$ и $t_{2}$ с достаточно большой разностью $t_{2}-t_{1}$. Более того, траектория эргодична, т.к. указанный интеграл равен среднему 
от функции $f(X(t))$, взятому по ансамблю промежуточных метастабильных участков при фиксированном $t$. Вероятностное распределение значений $X(t)$ на этих участках естественно принять в качестве стационарного вероятностного распределения значений процесса $X^{*}(t)$. Оно изображено на рис. 7 .

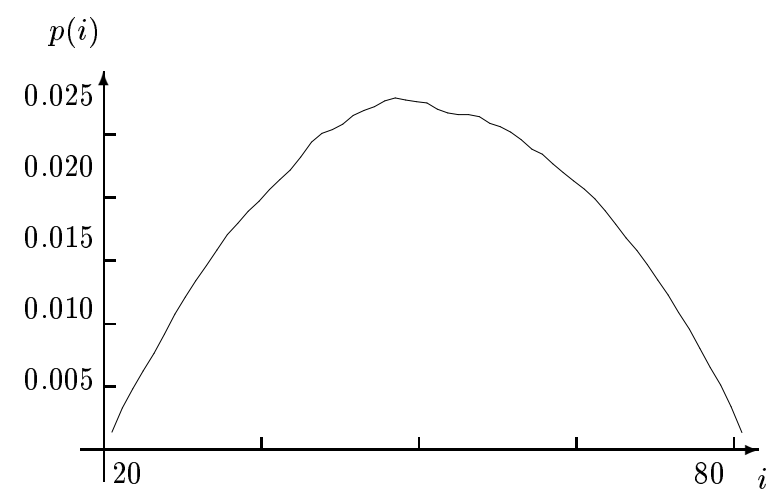

Рис. 7. Стационарное вероятностное распределение процесса $X^{*}(t)$.

Стационарное вероятностное распределение процесса $X^{*}(t)$ может использоваться как аналог меры Гиббса для промежуточного метастабильного состояния.

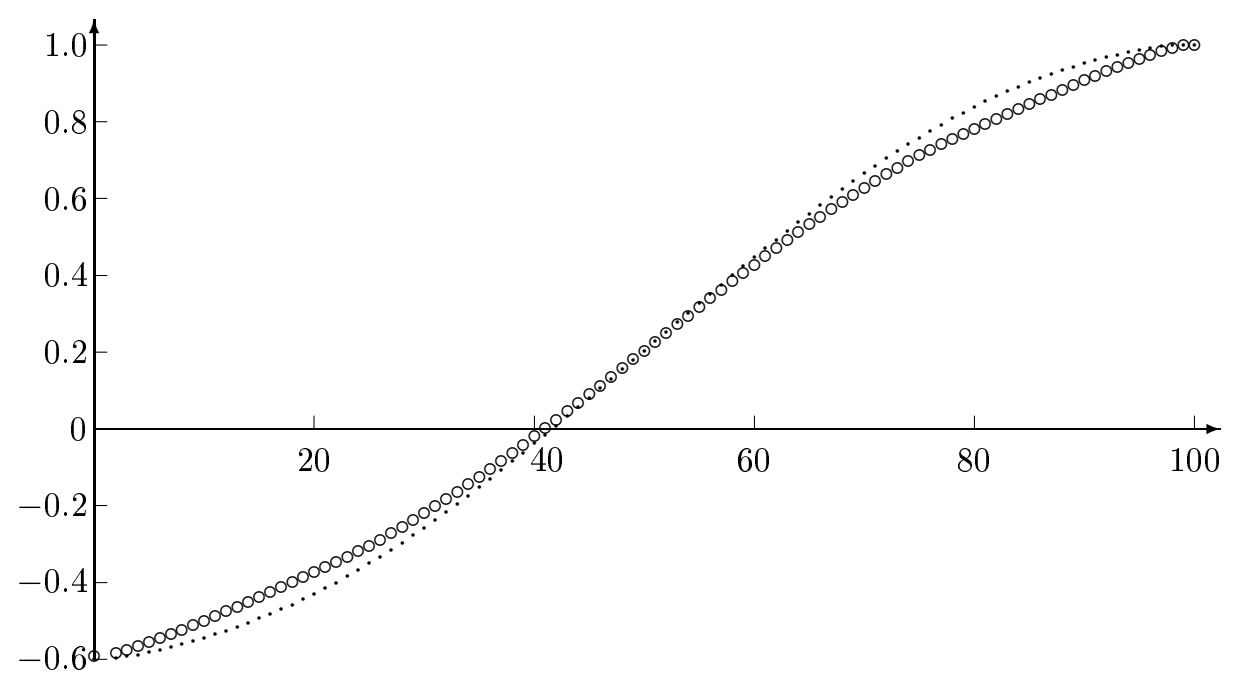

Рис. 8. Собственные значения матрицы вероятностей перехода при $T=T_{\mathrm{cr}}$.

3.3. Спектр матрицы переходных вероятностей. В работе [9] был сделан вывод о том, что метастабильное состояние может сушествовать в случае, если в спектре 
оператора эволюции есть щель. В нашей модели аналогом оператора эволюции является матрица вероятностей перехода $P$. Мы численно решили задачу о ее собственных

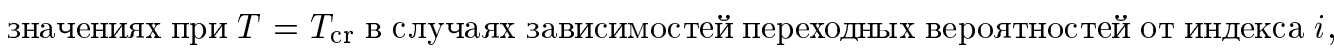
представленных на рис. 2 и 5. Найденные таким образом спектры приведены на рис. 8 (где белые и черные кружки - соответствуюшие спектры $P$ ). По оси Оy отложены собственные значения, а по оси $\mathrm{O} x$ - их формальная нумерация. Шели в спектрах не обнаружены.

\section{4. ЗАКЛЮЧЕНИЕ}

Фазовый переход между состояниями $A$ и $B$ с мерами Гиббса $\mu^{A}$ и $\mu^{B}$ описан как нестационарный марковский процесс с начальным распределением $\mu^{A}$, выходящий на стационарный режим с распределением $\mu^{B}$. Предложены простые формулы для переходных вероятностей этого процесса, с помощью которых удается воспроизвести основные черты фазового перехода. В численных экспериментах выявлены участки выборочных траекторий, интерпретируемые как метастабильные состояния. Найдены метастабильные состояния двух типов - состояние ожидания и промежуточное состояние. Время пребывания в промежуточном состоянии много меньше, чем в состоянии ожидания. Другими словами, промежуточное состояние является довольно редким явлением. Тем не менее удалось наблюдать достаточное количество траекторий в промежуточном состоянии и выяснить, что они являются отрезками траекторий некоторого стационарного процесса. Траектории в состоянии ожидания тоже обладают этим свойством.

Предложенная в данной работе модель фазового перехода получилась простой, поскольку использовано описание системы на промежуточном уровне между микроскопическим и макроскопическим. То, что удалось воспроизвести качественные закономерности фазового перехода, показывает, что такой подход может быть полезным. Однако количественное описание неравновесных систем с помощью марковских случайных процессов наталкивается на трудность выбора матрицы переходных вероятностей. Использованное в данной работе предположение о том, что $p_{i, j}=0$ при $|i-j|>1$, кажется, должно быть справедливо всегда, когда рассматриваются переходы за короткое время. В таком приближении конкретные значения вероятностей $p_{i, i}, p_{i, i+1}$ и $p_{i, i-1}$ можно найти, если учесть их структуру, определяемую формулами (3) и (4), и подобрать $K(i h)$ так, чтобы получились правильные равновесные распределения при различных значе-

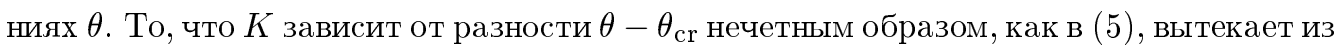
обших соображений о тенденциях при фазовом переходе.

Численные эксперименты проводились по аналогии с тем, как можно было бы изучать свойства метастабильных состояний в натурных экспериментах. Предположим, например, что требуется измерить плотность переохлажденного пара. Понятно, что из многих попыток получить переохлажденный пар большинство окажется неудачным. В наших численных экспериментах такие неудачные попытки представлены траекториями, быстро покидающими зону ожидания. Они должны быть исключены из статистических данных, а по остальным траекториям можно судить о свойствах переохлажденного пара. Отбор траекторий означает, что вместо математических ожиданий мы 
пользуемся условными математическими ожиданиями, т.е. вычисления средних выполняются при условии, что траектории находятся в зоне метастабильности длительное время. Поскольку такие траектории очень похожи на траектории некоторого стационарного процесса, вероятностное распределение этого процесса предлагается использовать как аналог меры Гиббса для метастабильного состояния.

Благодарности. Авторы благодарны профессору Л. Шульману за полезные дискуссии. Работа выполнена при поддержке Российского фонда фундаментальных исследований (грант № 96-15-96208).

\section{Список литературы}

[1] G. Parisi, Y. Wu. Sci. Sin. 1981. V. 24. P. 483; G. Parisi. Phys. Lett. B. 1983. V. 131. P. 393.

[2] J. R. Klauder. Acta Phys. Aus. Suppl. 1983. V. 25. Р. 53; A. А. Мигдал. УФН. 1986. Т. 149. С. 3; А. И. Кириллов. ТМФ. 1995. Т. 105. С. 179.

[3] А.И. Кириллов. УМН. 1994. Т. 49. С. 43.

[4] A. I. Kirillov. Found. Phys. 1997. V. 27. № 12. P. 1701.

[5] E. Nelson. Phys. Rev. 1966. V. 150. P. 1079.

[6] A. I. Kirillov. Phys. Lett. A. 1994. V. 195. P. 277.

[7] Л. Д. Ландау, Е. М. Лифиич. Статистическая физика. Часть 1. М.: Наука, 1995.

[8] О.И. Завьялов, М. Каненага, А.И. Кириллов, В. Ю. Мамакин, М. Намики, И. Охба, Е. В. Поляченко. ТМФ. 1996. Т. 109. С. 175.

[9] B. Gaveau, L. S. Schulman. J. Phys. A. 1987. V. 20. P. 2865.

[10] J.D. Gunton, M. Droz. Introduction to theory of metastable and unstable states. Berlin: Springer, 1983. 